Check for updates

Cite this: RSC Adv., 2019, 9, 20715

Received 5th May 2019

Accepted 27th June 2019

DOI: 10.1039/c9ra03340h

rsc.li/rsc-advances

\title{
Sulfaguanidine nanofiltration active layer towards anti-adhesive and antimicrobial attributes for desalination and dye removal $\uparrow$
}

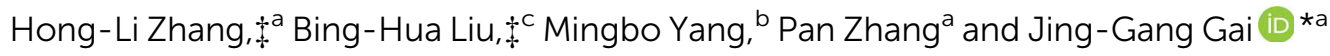

A novel sulfaguanidine (SG)-modified polyamide thin-film composite (TFC) nanofiltration (NF) membrane was constructed by the strategy referred to as co-solvent assisted interfacial polymerization (CASIP), which involves the respective interfacial polymerization (IP) of piperazine (PIP) and SG with trimesoyl chloride (TMC) on porous polysulfone (PSf) supports. CASIP enables the formation of a defect-free thin dense active layer and favors higher water permeance up to $79.0 \mathrm{~L} \mathrm{~m}^{-2} \mathrm{~h}^{-1}$ with rejection above $98.3 \%$ for $\mathrm{Na}_{2} \mathrm{SO}_{4}$. The resulting PA membrane also demonstrates a high flux recovery ratio of nearly $98.9 \%$ to bovine serum albumin protein after being cleaned. More importantly, the current membrane shows excellent anti-adhesive and antimicrobial performances against Gram-negative Escherichia coli, Grampositive Bacillus pumilus LDS.33 and Aspergillus parasiticus JFS. This promises great potential application of the PA membrane for practical water/wastewater treatment. The prospect of using the co-solvent mediated SG-modified layer as a next-generation anti-fouling/antimicrobial membrane is very encouraging.
\end{abstract}

\section{Introduction}

Membrane-based liquid separation processes such as reverse osmosis (RO) and nanofiltration (NF), on account of their low energy consumption and unique separation capabilities, have been applied in a broad spectrum of fields, such as desalination, and purification of seawater, brackish water, municipal water and industrial wastewater. ${ }^{1-3}$ These currently advanced polymeric membranes, however, are inherently prone to fouling, biofouling in particular, which dramatically deteriorates membrane performance, thus increasing operational cost and shortening the service life of membranes. ${ }^{4-6}$ Since NF membrane technology has been widely adopted owing to its low cost, high energy efficiency and environmental friendliness, ${ }^{7}$ to design and construct a novel NF membrane which can perfectly integrate high permselectivity and excellent anti-biofouling performance is highly desirable for efficient desalination and wastewater treatment.

${ }^{a}$ State Key Laboratory of Polymer Materials Engineering, Polymer Research Institute, Sichuan University, Chengdu, Sichuan 610065, China. E-mail: gaijinggang@scu.edu. cn; Fax: +8628 85402465; Tel: +862885467166

${ }^{b}$ College of Polymer Science and Engineering, State Key Laboratory of Polymer Materials Engineering, Sichuan University, Chengdu, Sichuan 610065, P. R. China

'Laboratory of Molecular Biology, College of Medicine, Chengdu University, Chengdu 610106, P. R. China

$\dagger$ Electronic supplementary information (ESI) available. See DOI: $10.1039 / \mathrm{c} 9 \mathrm{ra} 03340 \mathrm{~h}$

\$ These authors contributed equally to this work and should be considered co-first authors.
It is believed that membrane biofouling starts with the initial physical adsorption or deposition of organic macromolecules on membrane surface, followed by the adhesion of suspended microbes and eventually colonization by the irreversibly adhered microbes. ${ }^{6}$ Thus, the inhibition of membrane biofouling can be mainly classified into two approaches. One is to resist the macromolecules adsorption and microbes adhesion by constructing a surface with fouling resistance or fouling release property. ${ }^{8-10}$ The other is to control microbes colonization using the bactericidal tactic. ${ }^{11,12}$ To address the intractable biofouling problem, various strategies have been employed to avoid the attachment of macromolecules or suppress the growth and multiplication of microbes.

Membranes with the fouling resistance or fouling release tactic have been reported. ${ }^{13,14}$ Zwitterionic or amphiphilic polymers were grafted or coated onto the membrane surface to obtain a hydrophilic and low surface energy structure. ${ }^{15,16}$ The polymer with hydrophilic groups facilitates the formation of a compact hydration layer via electrostatic interactions and hydrogen bonds, which can sufficiently prevent macromolecules and microbes from adhesion onto membrane surface, reducing the irreversible membrane fouling. ${ }^{16}$ The low surface energy structure can minimize the intermolecular forces between foulants and membrane, thereby mitigate the reversible membrane fouling extent. ${ }^{14}$

To further inhibit the membrane biofouling, the bactericidal strategy is highly necessary. It serves as a "safeguard" with the aim of killing the adhered microbes by antimicrobial agents. Recently, considerable efforts have been devoted to developing 
novel antimicrobial agents to inhibit membrane biofouling, such as silver/copper nanoparticles, graphene oxides, and polymers with quaternary ammonium $\left(\mathrm{N}^{+}\right)$group. ${ }^{17-20}$ Unfortunately, due to the release-based mechanism, these toxic heavy metal nanoparticles are uncontrollably leached from membrane surface, rendering a regulatory risk in environment system. Also, bacterial strains with silver-resistance are emerging because of its extensive use. ${ }^{21}$ Moreover, many reported modification procedures may not be practical for industrialization. Physically applied coatings are more easily exfoliated. They would eventually lose antimicrobial activity during the long-time operational process. ${ }^{22}$ The newly developed covalent tethering method well maintains the stability of functional layer; however, it is time-consuming and its working process requires complex synthetic conditions. ${ }^{\mathbf{1 1 , 2 1 , 2 3 , 2 4}}$ Further, the complicated modification processes tend to deteriorate either membrane flux or rejection performance. ${ }^{8}$ Therefore, the biofouling inhibition of membrane surface via a more efficient, time-saving and benign approach that would offer superior permselectivity and anti-biofouling properties remains an alluring goal and needs further exploration.

Polymers containing guanidine or biguanidine groups have attracted substantial interest and proved to be effective cationic antimicrobial and biocidal agents. ${ }^{25,26}$ The guanidine-based polymers have shown outperforming properties over other antimicrobials, such as the exceptional broad-spectrum antimicrobial activity, excellent biocide efficiency and low toxicity to mammalian, thus enjoy increasing applications in industry, therapy and the clinic. ${ }^{27,28}$ The antibacterial activity of polymeric guanidine is ascribed to the progressive interaction between the cationic guanidine and the negatively charged groups including phospholipids, proteins and lipopolysaccharides on the bacterial cell membrane surface. The attraction immediately disrupts or imposes a charge imbalance, ${ }^{29}$ which would consequentially impel the breakdown of cells membrane, resulting to the leaching out of cellular content and the subsequent death of bacteria. ${ }^{30-32}$ There are some reports about incorporating polyhexamethylene guanidine hydrochloride (PHGH) onto the water-treatment membrane surface to improve the antibiofouling property. ${ }^{33,34}$ Although it is a promising antibacterial agent, the abundant consumption of high-cost $\mathrm{PHGH}$ and the insufficient quantity of hydrophilic groups in $\mathrm{PHGH}$ are unfavorable for the construction of the antifouling or antimicrobial surface, thereby strangling the anti-biofouling property of PHGH. Very recently, Zhao et al. ${ }^{35}$ modified a RO membrane with polydopamine immobilization by grafting a guanidinebased polymer, poly(guanidine-hexamethylenediamine-PEI) synthesized by the polycondensation of guanidine hydrochloride $(\mathrm{GH})$, 1,6-hexamethylenediamine and polyethyleneimine (PEI), to obtain excellent antifouling and antibacterial performances, but it is also not very hard to note its complicated synthesis process and the unsatisfactory permseletivity of GHfunctionalized membrane. Previously, we have proposed the fabrication of high water permeability and biofouling resistance PA-TFC NF membranes via a convenient and time-efficient strategy of pouring separately a series of small-molecule guanidine aqueous solutions including $\mathrm{GH}$, triamineguanidine hydrochloride (TAGH) and sulfaguanidine (SG) onto the surface of freshly prepared piperazine (PIP)trimesoyl chloride (TMC) membrane which was interfacially polymerized on the PSf ultrafiltration support membrane, namely, the second interfacial polymerization (SIP). ${ }^{36}$ Guanidine was covalently attached on the membrane surface through chemical reactions between the remaining acyl chloride group on the nascent PA membrane surface and the secondary guanidine amine monomers. Unfortunately, it is due to the poor solubility of SG dissolved in water phase that the rejection of SG-modified membrane is only $95.3 \%$ when the flux reaches to $79.0 \mathrm{~L} \mathrm{~m}^{-2} \mathrm{~h}^{-1}$ for $\mathrm{Na}_{2} \mathrm{SO}_{4}$ solution, which restricts its widespread applicability.

Herein, we design a novel SG-modified polyamide membrane with desirable seperating layer and nanopores size by choosing an appropriate co-solvent (acetone) containing the completely dissolved SG as the second amine monomer, then adding into the polar water phase. We refer to this polymerization approach as the co-solvent assisted second interfacial polymerization (CASIP) (Fig. 1). Interestingly, a narrow miscibility zone will be formed in the hexane/acetone/water system when the appropriate content of acetone is added into solution. ${ }^{37,38}$ In contrast to the hexane/water binary mixture with a great immiscibility gap over its entire composition range, the ternary system generates a wider liquid-liquid region, ${ }^{39}$ as depicted in Fig. 1. As a result, the formed polymeric networks can be fine-tuned by controlling the IP reaction zone. So far, various types of chemical additives and different techniques were utilized in the monomer-containing solutions to control the IP reaction and thus improve the permeation properties of the synthesized membranes. ${ }^{\mathbf{4 0 - 4 2}}$ Besides, the membrane properties and performance are optimized and systematically investigated in terms of physicochemical properties, separation performance for different inorganic salt, PEG and dye solutes, long-term stability, fouling resistance to bovine serum albumin (BSA) as well as anti-adhesive and antimicrobial activities against Gram-negative Escherichia coli (E. coli), Gram-positive Bacillus pumilus (B. pumilus) LDS.33 and Aspergillus parasiticus (A. parasiticus) JFS.

\section{Experimental section}

\subsection{Materials}

All chemicals were used as received: 1,3,5-benzenetricarboxylic chloride (TMC), (98\%, Alfa), piperazine (PIP) (99\%, anhydrous, Alfa), BSA (96\%, Sigma-Aldrich). Sulfaguanidine (SG) (98\%, TCI). Dyes including methyl orange (MO), chrome black $\mathrm{T}$ (CBT), rhodamine B (RB), congo red (CR), methyl blue (MB) were purchased from Aladdin Reagent Company (Shanghai, China). Neutral solutes (PEG200, PEG400, PEG600 and PEG1000) and all other reagents such as $n$-hexane, $\mathrm{NaOH}$, $\mathrm{Na}_{2} \mathrm{SO}_{4}, \mathrm{MgSO}_{4}, \mathrm{NaCl}, \mathrm{CaCl}_{2}$ were of analytical grade and supplied by Kelong Chemical Reagent Factory (Chengdu, China). All chemicals mentioned above were used without further purification. Flat-sheet nonwoven-reinforced polysulfone (PSf) ultrafiltration membranes [molecular weight cutoff $(\mathrm{MWCO})=60$ 000] as supports were provided by Beijing 


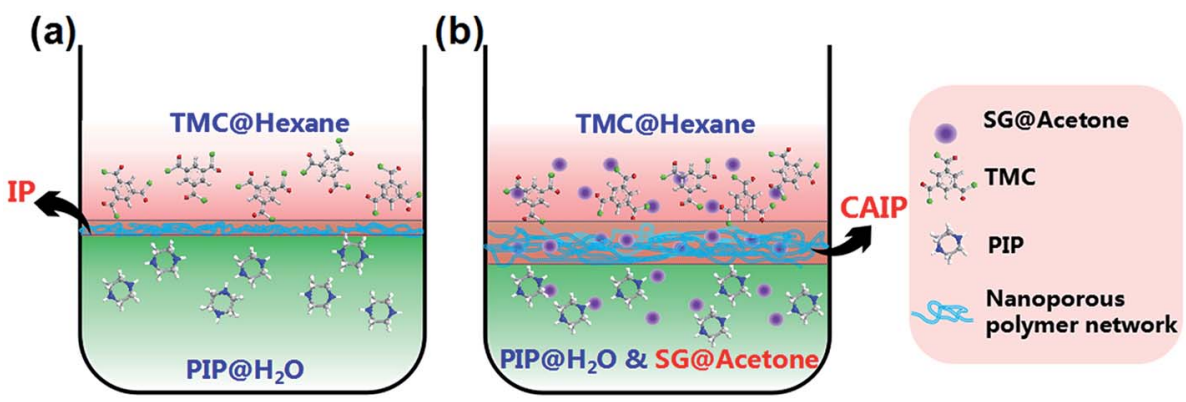

Fig. 1 Schematic diagram comparison of the conventional IP (a) and a novel CAIP (b). Reactant TMC dissolved in hexane phase and PIP dissolved in aqueous solution to react the first IP; SIP was conducted by dissolving the SG in acetone solution then a certain amount of aqueous solution with the $\mathrm{pH}$ of 12 was added with stirring, which diffuses toward one another and reacts to form polymeric networks.

Haiqingyuan Technology Co. Ltd. Deionized (DI) water with the conductivity less than $3 \mu \mathrm{sm}^{-1}$ was produced by water distiller.

\subsection{Membrane fabrication}

The SG-modified PA membrane was synthesized on porous PSf supports through a developed CASIP strategy. The preparation process is illustrated in Fig. 2. Typically, the pristine NF membrane was fabricated via the conventional IP of PIP and TMC. First, 0.35 wt\% PIP aqueous solution with a $\mathrm{pH}$ of 12 adjusted using $\mathrm{NaOH}$ was poured onto a top of PSf membrane clamped in a polyfluortetraethylene frame. Then the excess aqueous solution was drained off the surface after $2 \mathrm{~min}$. The soaked support was dried at room temperature until the surface appears dull and dry. Subsequently, the hexane solution containing $0.3 \mathrm{wt} \% \mathrm{TMC}$ was gently poured on the PIP saturated PSf support for $1 \mathrm{~min}$, the excess TMC solution was decanted. Further CASIP modification was carried out by pouring the acetone/water mixed solutions of SG with stirring onto the nascent PA membrane surface to react with residual acyl chloride groups. After $2 \mathrm{~min}$ of residence time, the excess solution was removed, and the membrane was dried in air for $3 \mathrm{~min}$ then subjected to $50{ }^{\circ} \mathrm{C}$ heat treatment for $15 \mathrm{~min}$, which results in the formation of the CASIP SG-modified PA membrane over the support. Finally, PA membrane samples were rinsed with DI water and stored in $\mathrm{NaHSO}_{3}(1 \mathrm{wt} \%)$ aqueous solution until use.
The acetone/water mixed solutions of SG was prepared as follows. First, an amount of SG was completely dissolved in $40 \mathrm{~mL}$ acetone solution and the $\mathrm{pH}$ values of $20 \mathrm{~mL}$ DI water was adjusted to 12 with $\mathrm{NaOH}$, then the two solutions were mixed in a stirred system. Here, we define the fabricated membranes as SG- $X$, where $X$ refers to the SG amount ( $\mathrm{g}$ ) added, thereby being donated as SG-0, SG-0.05, SG-0.1, SG-0.25, SG-0.3, SG-0.35, SG0.4 , SG-0.5.

\subsection{Membrane characterization}

All membrane samples were washed thoroughly with DI water and then dried in an oven $\left(30^{\circ} \mathrm{C}, 24 \mathrm{~h}\right)$ before all tests. Fourier transform infrared spectroscopy (FT-IR) and X-ray photoelectron spectroscopy (XPS) were employed to analyze the chemical structures of the PA active layers prepared by the CASIP and IP processes. FT-IR spectra were recorded on a Nicolet 380 spectrometer with an attenuated total reflectance (ATR) unit. XPS was performed on an XSAM800 spectrometer (Kratos, England). The surface morphologies of PA layers were characterized by field-emission scanning electron microscopy (FE-SEM, NOVA NANAOSEM 450, FEI, USA) and atomic force microscopy (AFM, Asylum Research MPD-3d). The surface root mean square (RMS) roughness of membranes was estimated from the threedimensional images of $5 \mu \mathrm{m} \times 5 \mu \mathrm{m}$ area using a tapping mode in air. FE-SEM was also employed to analyze the cross-

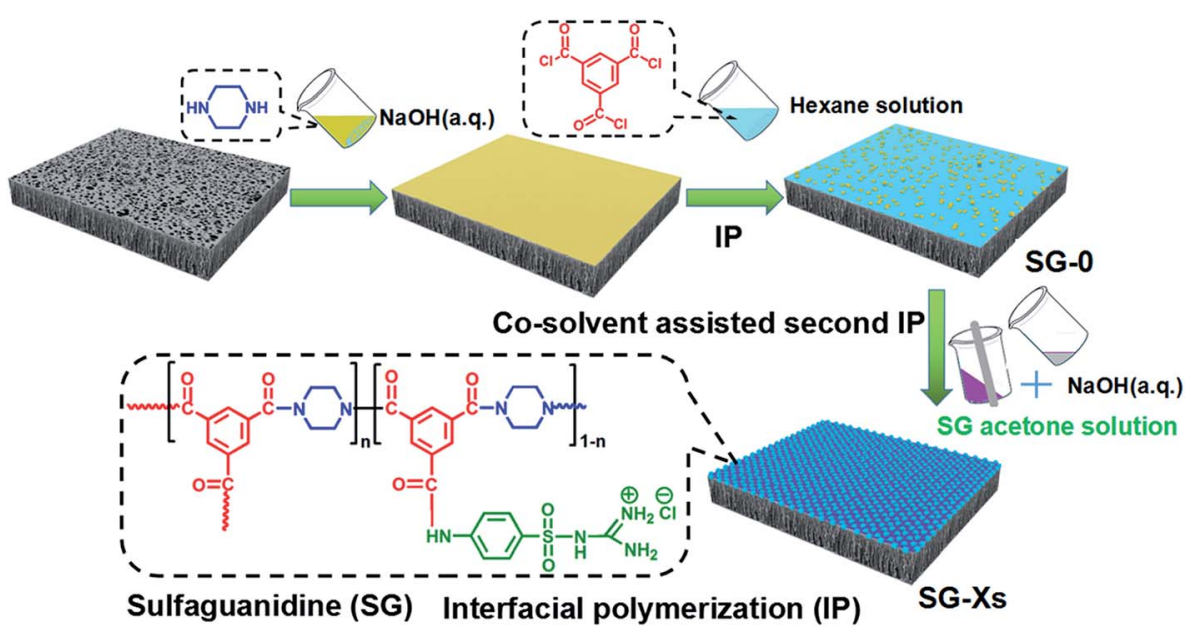

Fig. 2 Illustration of the fabrication process of CASIP SG-modified PA TFC membrane. 
sectional morphology of membranes, and the samples for crosssectional studies were obtained by fracturing membrane in liquid nitrogen. Cross-sectional morphology of membranes were observed by transmission electron microscopy (TEM, FEI Tecnai G2 F20, USA). For TEM analysis, the samples were prepared according to the reported method. ${ }^{43}$ Water CAs of membrane surfaces were determined with a goniometer (DSA30 drop shape analysis system, KRUSS) in sessile drop mode at room temperature. Each membrane was tested at least 5 times. $\mathrm{N}_{2}$ adsorption isotherms were measured by Quantachrome ASiQwin Instrument. Samples ( 200 mg) were used for $\mathrm{N}_{2}$ sorption analysis at liquid nitrogen temperature $(77 \mathrm{~K})$. Before the adsorption measurements, the samples were degassed in vacuum for $24 \mathrm{~h}$ at $80^{\circ} \mathrm{C}$. The zeta potential values of membrane surfaces were performed using an electrokinetic analyzer (SurPASS Anton Paar, GmbH, Austria) in a background electrolyte of $1 \mathrm{mM} \mathrm{KCl}$ solution over a $\mathrm{pH}$ range from 3 to 10 at room temperature.

\subsection{Nanofiltration experiments}

NF tests were performed in a cross-flow filtration apparatus with an effective area $(A)$ of $60 \mathrm{~cm}^{2}$. All performance tests were conducted at an operation pressure of 6 bar and a temperature of $25{ }^{\circ} \mathrm{C}$. Data were collected until steady state was reached, typically after half an hour. Water flux $\left(\mathrm{L} \mathrm{m}^{-2} \mathrm{~h}^{-1}\right)$ was calculated from the amount of the collected permeate $(V)$ over a certain time intervals $\Delta t$ as given by $V / A \Delta t$. The rejection $R(\%)$ was determined from the solute concentration in the feed $\left(C_{\mathrm{f}}\right)$ and the permeate $\left(C_{\mathrm{p}}\right)$ and calculated using the eqn (1):

$$
R=\left(1-\frac{C_{\mathrm{p}}}{C_{\mathrm{f}}}\right) \times 100 \%
$$

The concentration of $\mathrm{Na}_{2} \mathrm{SO}_{4}, \mathrm{MgSO}_{4}, \mathrm{NaCl}$ and $\mathrm{CaCl}_{2}$ aqueous solutions in the permeate $\left(C_{\mathrm{p}}\right)$ and feed $\left(C_{\mathrm{f}}\right)$ was evaluated using a conductivity meter (DDS-307, Shanghai INESA \& Scientific Instrument Co. Ltd, China). The concentrations of the PEG solutions were measured with a total organic carbon analyzer (TOC Analytik Jena 2100, Germany), and dyes concentrations were analyzed by a UV-vis spectrophotometer (UV3600). At least $20 \mathrm{~mL}$ solution was collected each time for the measurement. Permeation data were obtained with $2 \mathrm{~g} \mathrm{~L}^{-1}$ of $\mathrm{Na}_{2} \mathrm{SO}_{4}, \mathrm{MgSO}_{4}, \mathrm{NaCl}$ and $\mathrm{CaCl}_{2}, 200 \mathrm{mg} \mathrm{L}{ }^{-1}$ PEGs and $100 \mathrm{mg} \mathrm{L}^{-1}$ dyes in DI water as the feeds.

\subsection{Membrane fouling test}

Membrane anti-fouling property was assessed with model foulant of BSA at $25^{\circ} \mathrm{C}$ and 6 bar. The system was stabled with DI water for $1 \mathrm{~h}$ at an operating pressure of 6 bar. Then $\mathrm{Na}_{2} \mathrm{SO}_{4}$ aqueous solution $\left(2 \mathrm{~g} \mathrm{~L}^{-1}\right)$ was filtrated for $5 \mathrm{~h}$, and the water flux was monitored at fixed time intervals and recorded as $J_{0}$. Subsequently, BSA $\left(1 \mathrm{~g} \mathrm{~L}^{-1}\right)$ was instantaneously added to $2 \mathrm{~g}$ $\mathrm{L}^{-1} \mathrm{Na}_{2} \mathrm{SO}_{4}$. Finally, the membrane was washed with DI water for $1 \mathrm{~h}$. The filtration tests were operated with 2.5 cycles. The membrane fouling extent was assessed by the flux decline rate after fouling (FFD) and flux recovery rate after cleaning (FFR), which are the measured water flux normalized by the initial value. The FFD and FFR were calculated with eqn (2) and (3):

$$
\begin{aligned}
& \mathrm{FFD}=\left(1-\frac{J_{1}}{J_{0}}\right) \times 100 \% \\
& \mathrm{FFR}=\left(1-\frac{J_{2}}{J_{0}}\right) \times 100 \%
\end{aligned}
$$

where $J_{0}, J_{1}$ and $J_{2}$ correspond to the initial permeate flux, permeate flux at the end of fouling and permeate flux after cleaning, respectively.

\subsection{Membrane biofouling test}

2.6.1 Anti-adhesion property. We selected $\mathrm{G}^{-}$E. coli $\mathrm{K} 12$ (CGMCC 1.365), $\mathrm{G}^{+}$B. pumilus LDS33 (CCTCC M 2013179) and A. parasiticus JFS (CCTCC M 2013662) as model microorganisms to examine the anti-adhesive property of SG-Xs. The $E$. coli and B. pumilus strains were cultured in Luria-Bertani (LB) broth solution at $37{ }^{\circ} \mathrm{C}$ for 24 hours. The $E$. coli suspension with the

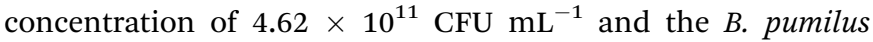
suspension of $5.94 \times 10^{10} \mathrm{CFU} \mathrm{mL}{ }^{-1}$ were obtained using the plate count method on the LB plate $\left(37^{\circ} \mathrm{C}, 24 \mathrm{~h}\right)$. Similarly, the A. parasiticus strain was cultured for 24 hours in Potato Dextrose Agar (PDA) solution at $30{ }^{\circ} \mathrm{C}$ and the concentration of $A$. parasiticus suspension was $3.3 \times 10^{9} \mathrm{CFU} \mathrm{mL}^{-1}\left(30^{\circ} \mathrm{C}, 24 \mathrm{~h}\right)$. After culturing, the $100 \mu \mathrm{L} \mathrm{E}$. coli and B. pumilus suspensions were respectively inoculated to the test tube containing $10 \mathrm{~mL} \mathrm{LB}$ broth solution with four replicate SG-0 and SG-0.3 membrane pieces $(\varnothing 2.0 \mathrm{~cm})$, subsequently culturing for $24 \mathrm{~h}$ at $37^{\circ} \mathrm{C}$. Next, the membranes were taken out from the tube and were thoroughly rinsed to collect the cells. Then the collected suspensions were diluted with sterilized water, and the diluents (100 $\mu \mathrm{L}$ ) were plated on the LB plate, incubating for $24 \mathrm{~h}$ at $37^{\circ} \mathrm{C}$. The viable colonies on the plate were counted, and the results are expressed as colony forming units per membrane area (CFU $\left.\mathrm{mL}^{-1}\right)$. The A. parasiticus suspension was treated with the same method in PDA medium, and the temperature was controlled at $30{ }^{\circ} \mathrm{C}$. The data provided are average values from three experiments of each membrane type.

2.6.2 Antimicrobial property. For the antimicrobial activity of membrane, we studied the growth status of three model microbes on to it. The sterilized water of $10 \mathrm{~mL}$ and six replicate membrane pieces $(\varnothing 1.0 \mathrm{~cm})$ were added in a test tube. Then the $150 \mu \mathrm{L} \mathrm{E}$. coli and B. pumilus suspensions were respectively inoculated, being cultured for $15 \mathrm{~h}$ at $37{ }^{\circ} \mathrm{C}$. Similarly, the $A$. parasiticus suspension was inoculated and cultured for $10 \mathrm{~h}$ at $30{ }^{\circ} \mathrm{C}$. The membranes were removed from the test tube, followed by the UV-vis analysis of suspensions at the absorption wavelength of $600 \mathrm{~nm}$.

2.6.3 Nanofiltration property after biofouling. To investigate the impact of biofouling on permeation performance, the water flux of the SG-0 and SG-0.3 membranes after $48 \mathrm{~h}$ incubation of microbial cultures (harsh conditions described in Section 2.6.1) was examined with $2 \mathrm{~g} \mathrm{~L}^{-1} \mathrm{Na}_{2} \mathrm{SO}_{4}$ solution via crossflow filtrations. 


\section{Results and discussion}

\subsection{Membrane surface chemical composition}

Membrane surface chemical components were characterized by ATR-FTIR and XPS analyses, and the results are shown in Fig. 3. It was found that there appeared a new peak at about $1620 \mathrm{~cm}^{-1}$ for SG-0 and SG-0.3 membranes, which is ascribed to the stretching vibration of $\mathrm{C}=\mathrm{O}$ in amide groups, which revealed that interfacial reaction had successfully occurred and a PA layer was grown onto the PSf supports (Fig. 3a). ${ }^{\mathbf{4 4 , 4 5}}$ While there is no discernible difference ATR-FTIR spectra between SG-0 and SG-0.3 layers likely due to the overlapped peaks of $\mathrm{C}=\mathrm{N}$ stretching vibration $\left(1647 \mathrm{~cm}^{-1}\right)$ and already existing $\mathrm{C}=\mathrm{O}$ stretching vibration of PA. ${ }^{46}$ The eminent peaks of SG-0 and SG0.3 layers at $285.0 \mathrm{eV}, 400.0 \mathrm{eV}$ and $532.0 \mathrm{eV}$ were assigned to carbon ( $\mathrm{C}$ 1s), nitrogen ( $\mathrm{N}$ 1s) and oxygen ( $\mathrm{O}$ 1s), respectively (Fig. 3b). ${ }^{47}$ As depicted in Fig. 3c, two new peaks at $231.6 \mathrm{eV}$ and $168.0 \mathrm{eV}$ in SG-0.3 respectively ascribed to sulfur (S) $2 \mathrm{~s}$ and $\mathrm{S} 2 \mathrm{p}$ suggest the successful immobilization of SG. ${ }^{15}$ As can be seen from Table $\mathrm{S} 1, \dagger$ the $\mathrm{S}$-content on membrane surface increases with the amount of SG being increased from $0.05 \mathrm{~g}$ to $0.5 \mathrm{~g}$. The high-resolution XPS spectra for C 1s and N 1s of SG-0 and SG-0.3 PAs, which provide more detailed information of the chemical compositions of PA skin layer, are illustrated in Fig. 3d, e and f, $\mathrm{g}$, respectively. The $\mathrm{C} 1 \mathrm{~s}$ component peaks at binding energy of 284.2, 285.4 eV and 287.6 (Fig. 3d and f) are assigned to $\mathrm{C}=\mathrm{C} / \mathrm{C}-$ $\mathrm{C}, \mathrm{N}-\mathrm{C}=\mathrm{O}$ and $\mathrm{C}-\mathrm{N}$, respectively, ${ }^{48,49}$ while $\mathrm{N} 1$ s component peaks at $399.4 \mathrm{eV}$ and $399.9 \mathrm{eV}$ (Fig. 3e and g) are assigned to $\mathrm{N}-\mathrm{C}=\mathrm{O}$ and $\mathrm{C}-\mathrm{N}$, respectively. ${ }^{50}$ A novel peak at $398.2 \mathrm{eV}$ was clearly detected in SG-0.3 layer (Fig. $3 g$ ), ascribing to $\mathrm{N}-\mathrm{S}$ specie, and the peak component area ratio of $\mathrm{N}-\mathrm{S}$ on the SG-0.3 layer was $6.6 \%$. The fraction of $\mathrm{N}-\mathrm{S}$ on membrane surface increased from $1.9 \%$ (SG-0.05) to $12.2 \%$ (SG-0.5) (see Fig. S1†). Also, the peaks at $167.5 \mathrm{eV}(\mathrm{S}-\mathrm{N}-\mathrm{C})$ and $168.8 \mathrm{eV}(\mathrm{C}-\mathrm{S}=\mathrm{O})$ were observed on the SG-0.3 layer (Fig. 3h). ${ }^{51}$ These data confirmed that the SG monomer has been successfully introduced onto PSf supports.

\subsection{Membrane surface morphology and microporous structure}

Besides, the microscopy images highlight the advantage of CASIP tactic in creating more uniform and fairly dense skin layer with a relatively smooth surface. As Fig. 4a-f images illustrate, the nascent PA layer displays a nodular morphology that is typical of interfacially polymerized, semi-aromatic PA using TMC and PIP, ${ }^{50}$ whereas that of the CASIP SG-modified membranes has much more obvious grainy structure throughout the surfaces. Notably, the better-dispersed and most uniform granules covers the SG-0.3 surface (Fig. 4d). To further confirm the changes in morphology, AFM was employed to analyze the roughness of the surface. The AFM height and topological images of SG- $X$ s are depicted in Fig. $4 \mathrm{~g}-\mathrm{l}$ and $\mathrm{S} 3, \dagger$ respectively. It should be noted that the RMS values become gradually smaller, then increased after modification, and the
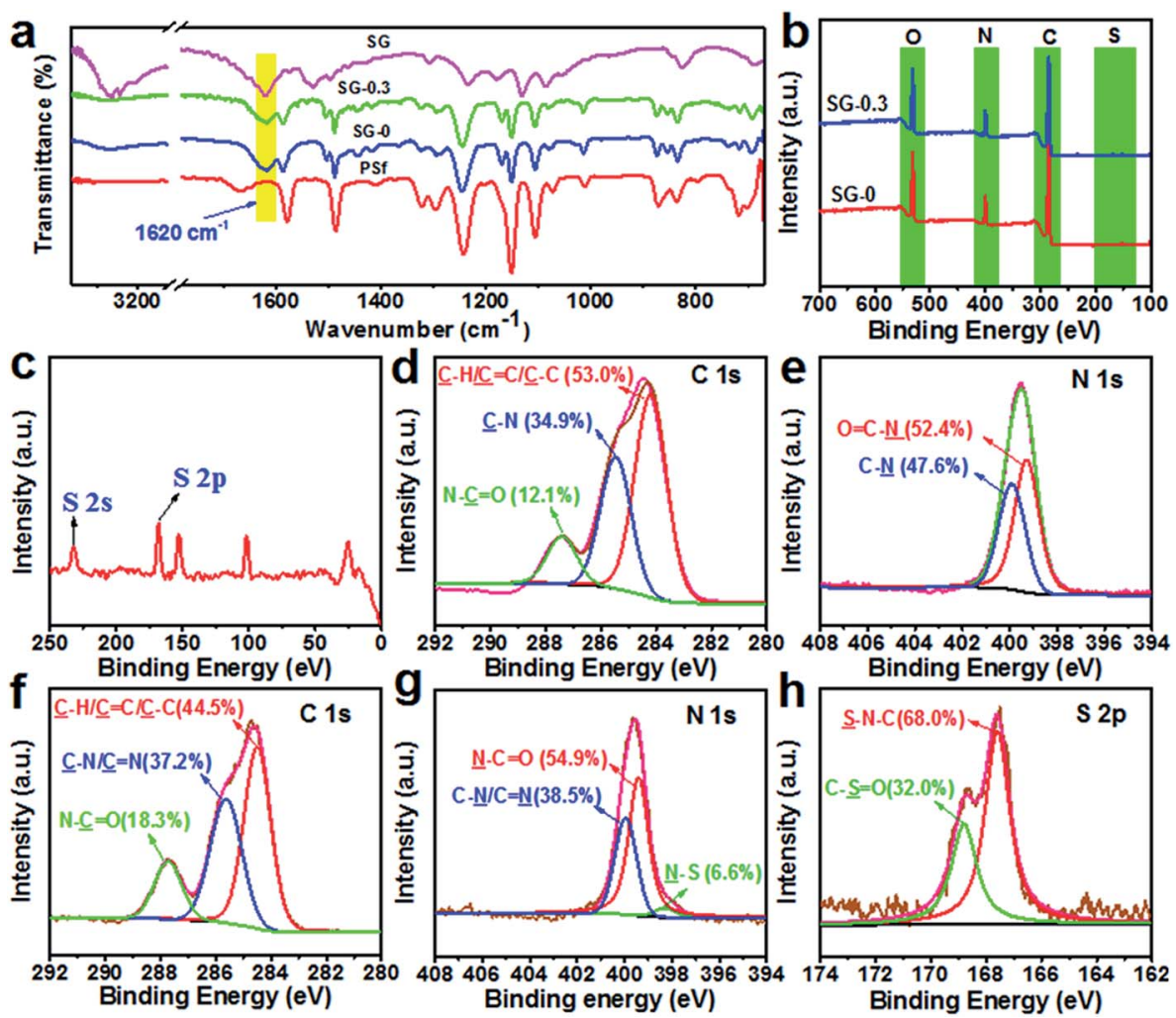

Fig. 3 (a) Comparison of ATR-FTIR spectra between PSf, SG, SG-0 and SG-0.3 membranes; (b) XPS survey spectra wide-scan for the SG-0 and SG-0.3 membranes and a larger version for SG-0.3 (c); C 1s (d) and N 1s (e) core-level spectra for SG-0 layer; C 1s (f), N 1s (g) and S 2p (h) corelevel spectra for SG-0.3 layer. 

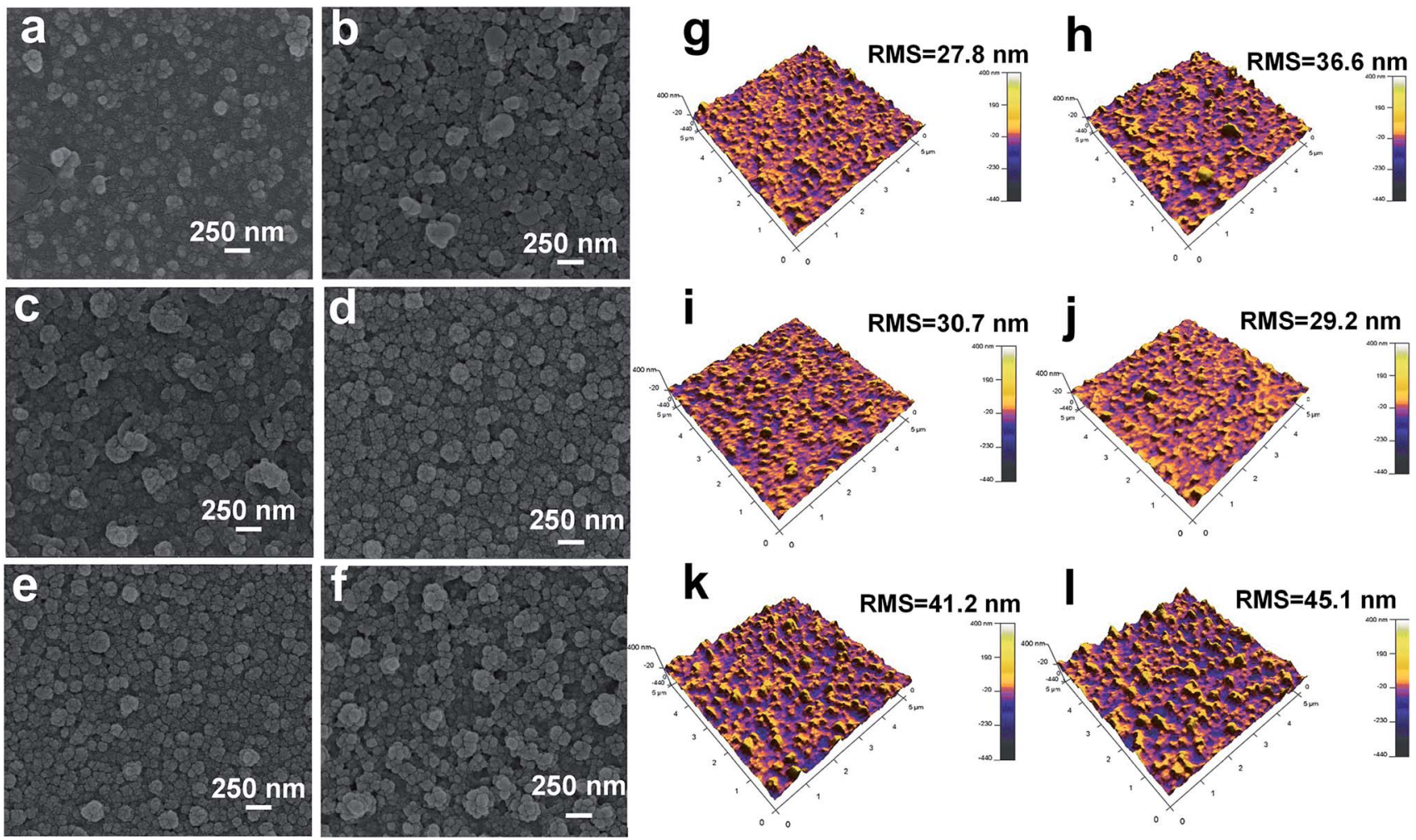

Fig. 4 The SEM surface (left) and AFM height (right) images of SG-0 (a/g), SG-0.05 (b/h), SG-0.1 (c/i), SG-0.3 (d/j), SG-0.4 (e/k) and SG-0.5 (f/l) membranes.

SG-0.3 skin layer has the minimum roughness value, in accordance with the SEM images. The improved smoothness is probably due to the covalently tethering of polymer chains of PIP-TMC with the SG layer to fill the valley structures on the pristine membrane surface. ${ }^{52}$ Moreover, the attachment of SG onto the nascent PA layer would not affect the depositions of foulants on membrane surface caused by surface roughness,$^{53}$ which plays a positive role for the membrane antifouling performance. To gain insight into the active layer morphology, the cross-sectional SEM and TEM images were obtained. As Fig. 5a, b, S4a and b $\mathrm{b}^{\mathrm{s}}$ show, the raw PA layer has the thickness of around $227 \mathrm{~nm}$, while the thicknesses of the SG-modified layers are $137-221 \mathrm{~nm}$. The slight decrease in thickness is due to the more compact and tightened polymer chains derived from the inherent structure of SG, as evidenced by the corresponding TEM images (Fig. $5 \mathrm{c}, \mathrm{d}, \mathrm{S} 4 \mathrm{c}$ and $\mathrm{d} \dagger$ ). ${ }^{54}$ Besides, the thinner dense layer structure might be caused by a narrow miscibility zone formed in the CASIP system, where the interfacial tension and solubility differences are greatly decreased, rendering the formation of a loose and thin polymer layer.

The gas sorption experiment has often been applied to analyze the porous properties of nanoscale organic polymer networks; therefore we conjectured that it should be a suitable method for the porosity analysis of the active layer of PA membranes containing nanometer-size pores. ${ }^{55,56}$ The microporous property of SG-0 and SG-0.3 membranes was probed by $\mathrm{N}_{2}$ adsorption/desorption experiment. The samples for the $\mathrm{N}_{2}$ sorption analysis were synthesized in a beaker according to the same IP recipe and procedure conditions for the formation of thin films of PA membranes at the solution interface, and thus could be considered structurally similar to the polymer networks structure composing the active layer of the respective $\mathrm{NF}$ membrane. ${ }^{57}$ As evident by Fig. $5 \mathrm{e}$, the $\mathrm{N}_{2}$ adsorption/ desorption isotherm of the SG-0.3 PA at low relative pressures $\left(P / P_{0}<0.01\right)$ exhibits steep nitrogen uptakes, indicating its microporous feature. ${ }^{55}$ However, a type H3 hysteresis loop in the sorption curve suggests the presence of some mesopores in the SG-0.3 polymeric network..$^{58}$ The mesoporous structure probably exists in the outer loose PA network structure formed later as the PIP monomer diffuses through the dense core layer during the IP process. ${ }^{57}$ As Fig. $5 \mathrm{f}$ illustrate, the pore size distribution (PSD) curves derived from the $\mathrm{N}_{2}$ adsorption isotherm using Saito-Foley (SF) method show the pore radius of SG-0 PA centering around $1.07 \mathrm{~nm}$, while larger pores with a primary radius of $1.18 \mathrm{~nm}$ are revealed from the PSD curve of SG-0.3 PA. According to the literatures already reported, the network pores or aggregate pores in NF membrane are in fact free volumes of polyamide matrix. Since the larger free volumes of PA matrix in the SG-0.3 network has been mainly ascribed to the wider miscibility zone, which leads to the formation of the much loose polymeric layer. Besides, the large molecular size and stiffening ring structure of SG possibly account for the increase of free volumes of polymeric networks. Such the microstructure features would greatly influence the subsequent separation performance for salt rejection and dye removal. 

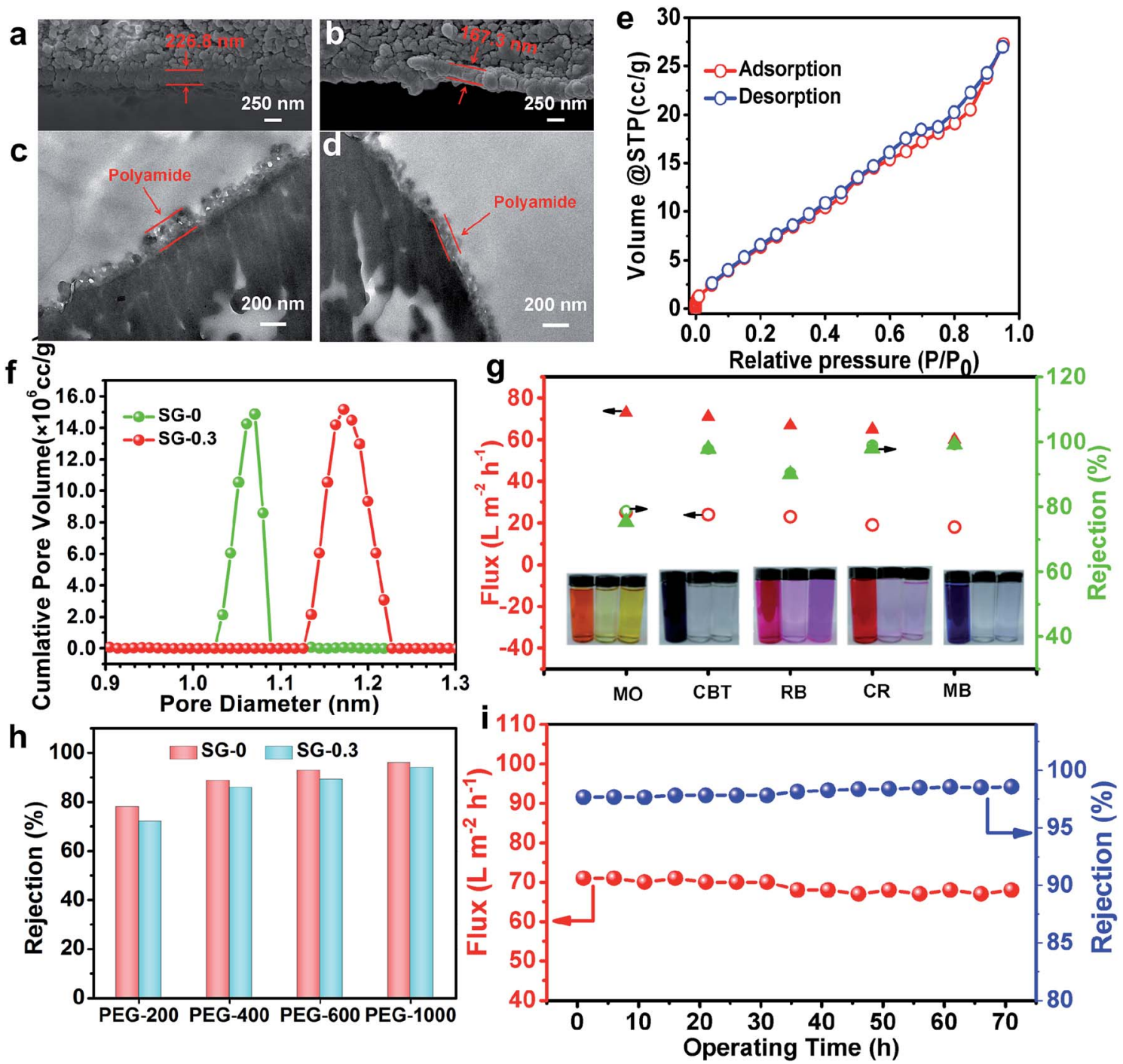

Fig. 5 The cross-sectional SEM images of SG-0 (a) and SG-0.3 (b) membranes, and the cross-sectional TEM images of SG-0 (c) and SG-0.3 (d) membranes; (e) nitrogen adsorption-desorption isotherm of the SG-0.3 membrane; ( $f$ ) the micropore size distribution curves of the SG-0 and SG-0.3 membranes; (g) NF performances of the SG-0 and SG-0.3 membranes for $100 \mathrm{mg} \mathrm{L}^{-1}$ dyes; (h) the rejections of the SG-0 and SG-0.3 membranes for $200 \mathrm{mg} \mathrm{L}^{-1}$ PEGs; (i) the stability test of the SG-0.3 membrane in a long-time NF for CBT.

\subsection{Membrane permselectivity properties for PEGs,} inorganic salts and dyes

Theoretically, the Stokes radius $\left(r_{\mathrm{s}}, \mathrm{nm}\right)$ and diameters $\left(d_{\mathrm{s}}\right)$ for PEG molecules at a given $M_{\mathrm{w}}\left(\mathrm{g} \mathrm{mol}^{-1}\right)$ (Table $\left.\mathrm{S} 2 \dagger\right)$ can be calculated with eqn (4): ${ }^{59}$

$$
r_{\mathrm{s}}=1.673 \times 10^{-2} M_{\mathrm{w}}^{0.557}
$$

Considering the steric and hydrodynamic hindrance effects of the pore on solute molecules, the relationship between the solute rejection ( $\left.R_{\text {theory }}\right)$ and $\eta\left(\eta=d_{\mathrm{s}} / d_{\mathrm{p}}\right.$, the ratio of solute diameter to pore diameter) can be fitted by the following expression: ${ }^{60}$

$$
R_{\text {theory }}=1-\left\{1-[\eta(\eta-2)]^{2}\right\} \exp \left(-0.714 \eta^{2}\right)
$$

The practical rejection of SG-0 and SG-0.3 active layers for PEG400 is about $88.64 \%$ and $85.89 \%$, respectively (Fig. 5h).
According to eqn (5), the pore diameters of the SG-0 and SG-0.3 layers were respectively found to be 1.329 and $1.389 \mathrm{~nm}$, which agree well with the values from SF model (Fig. 5f), suggesting the theoretical model can be adequately used for estimating the real diameter of "pore" in NF membrane. The MWCO of SG-0 and SG0.3 membranes calculated from eqn (4) and (5) under the rejection of $90 \%$ is about 417 and $451 \mathrm{Da}$, respectively, demonstrating nanofiltration characteristics. Accordingly, the model of physical sieving by nanoporous polymer network which is controlled by pore sizes dominates in the retention mechanism.

Water flux and salt rejection of the SG- $X \mathrm{~s}$ membranes were tested. For all SG-modified membranes, the permeating fluxes increase drastically, but level off at higher SG amount owing to the exhausting of acyl chloride groups and the blocked nanometer-size pores (Fig. 6a). ${ }^{36}$ To be specific, the flux of SG0.3 membrane for $\mathrm{Na}_{2} \mathrm{SO}_{4}, \mathrm{MgSO}_{4}, \mathrm{NaCl}$ and $\mathrm{CaCl}_{2}$ reached to 79, 72, 90, $83 \mathrm{~L} \mathrm{~m}^{-2} \mathrm{~h}^{-1}$, respectively, which are about 3.2-fold 


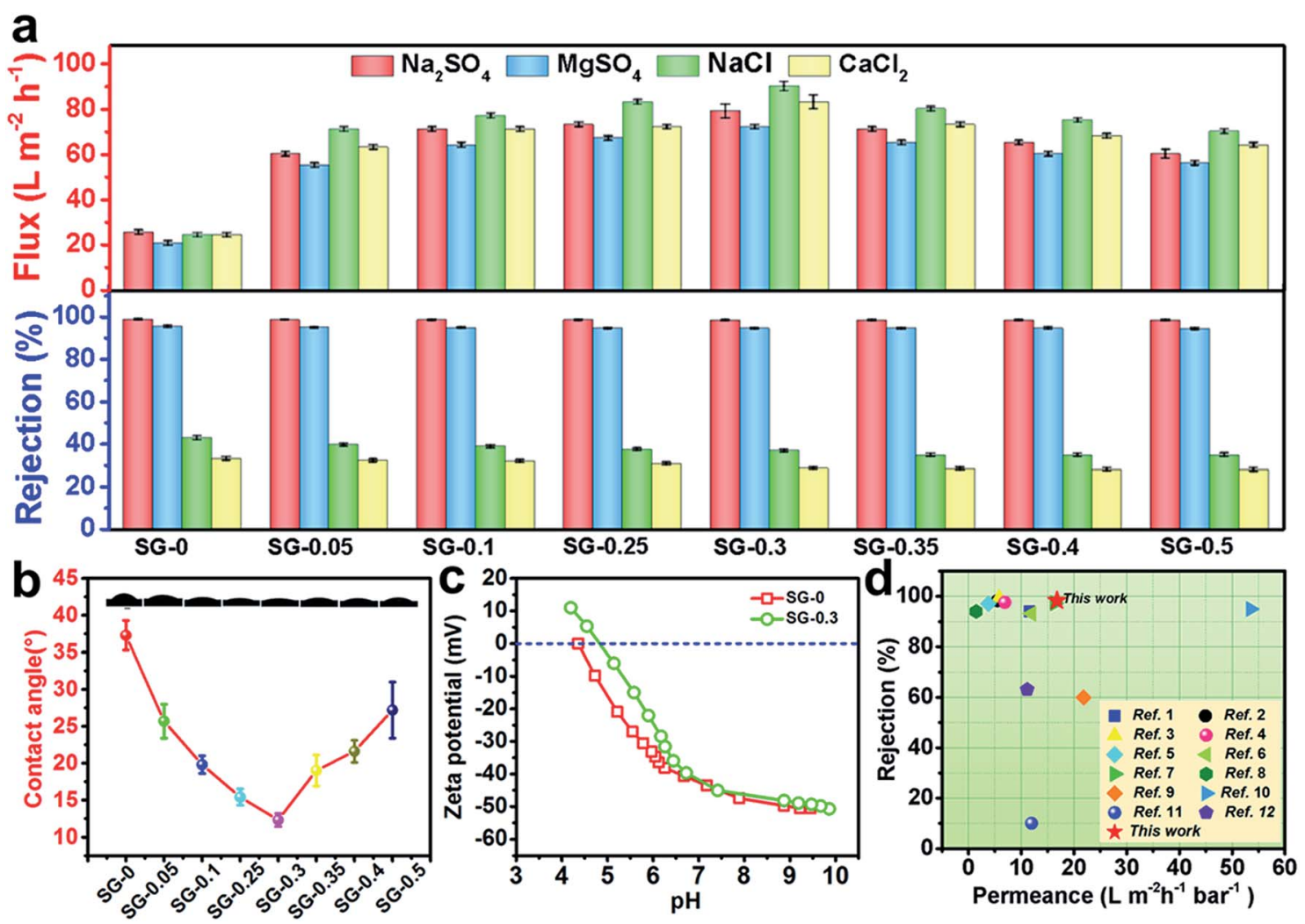

Fig. 6 (a) NF performances of SG-Xs for salts aqueous solution $\left(2 \mathrm{~g} \mathrm{~L}^{-1} \mathrm{Na}_{2} \mathrm{SO}_{4}, \mathrm{MgSO}_{4}, \mathrm{CaCl}_{2}, \mathrm{NaCl}\right)$ at $25^{\circ} \mathrm{C}$ and 6 bar; (b) water CAs of SG-XS; (c) zeta potentials of the SG-0 and SG-0.3 surface at various pH values; (d) summary of the separation performance of the state-of-the-art NF membranes reported recently in literature in consideration of permeance and rejection for $\mathrm{Na}_{2} \mathrm{SO}_{4}$ (for details see Table $\mathrm{S} 3 \dagger$ ).

comparable to that of the traditional interfacially polymerized membrane (SG-0, 25, 20, 24, 24 $\mathrm{L} \mathrm{m}^{-2} \mathrm{~h}^{-1}$ ), while remaining a reasonably high salt rejection simultaneously (Fig. 6a). The remarkable enhancement in permeance benefits from guanidino units with the unique water activating propensity and the "structure breaking" effect. The strong basicity of SG molecules in aqueous solution is fully ionized and consequently induces polarization to form a hydrated layer with strong water-binding ability, ${ }^{61,62}$ hence the hydrophilicity is enhanced and the water molecules penetrate through the SG-grafted active layer more readily. On the other hand, due to the unique "structure breaking" effect, large clusters of water molecules around the guanidino units could be changed into small clusters, which are highly helpful to enhance water permeability. ${ }^{63}$ Indeed, the remarkably improved hydrophilicity was confirmed by comparing the water contact angles (CAs) of different surfaces before and after modifications. The CAs of SG-0 and SG-0.3 membranes were $37.3^{\circ}$ and $12.3^{\circ}$, respectively (Fig. 6b). Importantly, the super-hydrophilic surface is beneficial for the reduction of membrane fouling caused by hydrophobic foulants. ${ }^{64}$ Furthermore, comparative to the interfacially polymerized analog, the SG-0.3 demonstrates slightly lower salt rejections $\left(98.6 \%\right.$ vs. $98.3 \%$ to $\mathrm{Na}_{2} \mathrm{SO}_{4}, 95.3 \%$ vs. $94.4 \%$ to $\mathrm{MgSO}_{4}, 42.7 \%$ vs. $36.7 \%$ to $\mathrm{NaCl}, 32.9 \%$ vs. $28.4 \%$ to $\mathrm{CaCl}_{2}$, respectively), which is mainly attributed to the lessened electrostatic repulsive effect between the multivalent anions $\left(\mathrm{SO}_{4}{ }^{2-}\right)$ and membrane surface (Fig. 6a). ${ }^{65}$ The zeta potential of PA layer decisively influences the solute rejection, hence we measured the zeta potential data over the $\mathrm{pH}$ range of 3.0-10.0. The surface of SG-0.3 features less negatively charged than the SG-0, which is consistent with the above mentioned conjecture regarding to the changes of salt rejection (Fig. 6c). These results signify that an NF membrane with unprecedentedly high water permeance and a satisfactorily high rejection salt rejection was achieved via the CASIP procedure. The filtration performance regarding permeance and salt rejection of SG-0.3 membrane is also compared with those of several commercial and other stateof-the-art NF membranes reported recently in literatures, ${ }^{7,16,47,48}$ (ESI ref. 1-8†) which are summarized in Fig. 6d. The comparison suggests that the NF performance of CASIP SG-modified membrane is, to a large extent, superior to all other membranes recently developed, including 2D nanomaterials membranes. ${ }^{7,16}$ Moreover, the CASIP procedure introduced in this work is straightforward with no post treatments in contrast to that of other relevant PA membranes like the timeconsuming zwitterionic membrane. The high-performance SGmodified membrane fabricated by the CAIP method also shows good reproducibility. The co-solvent mediated IP technique renders the creation of a defect-free, highlypermselectivity layer on porous supports in a controlled manner, and would potentially offer not only possibility but also new degrees of freedom in designing either NF or reverse osmosis membranes with excellent properties employing the poorly water-soluble amine monomers. 
Dyes are very common in our daily life, and a mass of dye wastewater are produced in many fields, such as textiles, packaging, pharmaceuticals, and cosmetics. These dyes aqueous waste can cause considerable environmental pollution and serious health hazards. ${ }^{66}$ To explore the application of the newly developed SG-modified membrane for dye wastewater treatment, retention measurements with solutions of MO, CBT, $\mathrm{RB}, \mathrm{CR}$ and $\mathrm{MB}$ were conducted. As Fig. $5 \mathrm{~g}$ verifies, in every case, colorless filtrate was obtained with a highly promising rejection rate for the both SG-0 and SG-0.3 membranes. For instance, the SG-0.3 membrane can reject CR $\left(M_{\mathrm{w}}=696.66 \mathrm{Da}\right)$ and $\mathrm{MB}\left(M_{\mathrm{w}}=\right.$ $799.80 \mathrm{Da})$ with rejection rates of $98.01 \%$ and $99.2 \%$, respectively. Strikingly, the permeation fluxes after modification are nearly 2-fold higher than that of the conventional NF membrane, meanwhile maintaining similar high rejection. It should be noted that the rejection rates of SG-0 and SG-0.3 active layers for $\mathrm{RB}\left(M_{\mathrm{w}}=479.02 \mathrm{Da}, c a .1 .20 \mathrm{~nm} \times 1.13 \mathrm{~nm}\right)$ are markedly lower than that of CBT $\left(M_{\mathrm{w}}=461.38, c a .1 .55 \mathrm{~nm}\right.$ $\times 0.88 \mathrm{~nm}$ ) with the similar molecule size. This is probably due to the difference of charge characteristic of dye solutions. Typically, the charge character of the active layer is a critical factor governing the solute transport through the charged PA membrane. Based on the Donnan exclusion theory, ${ }^{65}$ for the negatively charged SG-0 and SG-0.3 membrane, the negatively charged dye solutes should be better retained. Consequently, the rejection for dyes was contributed by synergistic physical sieving from nanometer-size pores and the electrostatic interaction between charged dyes and membrane surface. Additionally, our membrane shows the outstanding stability concerning water permeance and dye rejection (Fig. 5i) as its benefits from the merit of covalent grafting modification and its super-hydrophilicity. No appreciable decrease of permeating flux was observed over $71 \mathrm{~h}$ in continuous filtration, and the rejection rate for CBT remained constant (98.4\%). Overall, the CASIP SG-modified membrane shows extremely high permeability and reasonably high retention for salts and organic dyes, together with the outstanding stability, indicating its potential towards the efficient desalination and dye wastewater treatment.

\subsection{Membrane antifouling property}

To evaluate the antifouling performance, the relative water fluxes of the all fabricated membranes were monitored as a function of time during the filtration with the feed solution including BSA as a model foulant. Notably, the SG-0.3 membrane showed a lower ultimate flux decline $(\approx 13 \%)$ than the SG-0 membrane $(\approx 28 \%)$; after the washing process, the flux of SG-0 membrane recovered to $83.0 \%$ whereas that of SG-0.3 reached up to $98.9 \%$, demonstrating its superior antifouling ability (Fig. 7a). The good antifouling performance was confirmed by morphological results under SEM, shown in Fig. 7b-g. Unlike the SG-0 membrane, whose surface was significantly covered by BSA which cannot be removed by physical washing, there is less BSA adsorbed on the SG-0.3 surface and it was removed almost entirely from the membrane. This further reveals its better fouling resistance to BSA. The time-dependent relative water flux results of other prepared membranes were shown in Fig. S7. $\dagger$ With the increase of SG dissolved in aqueous phase, lower ultimate flux decline rates and higher flux recovery rates were observed, revealing the better antifouling. Membrane fouling depends closely upon the surface physicochemical properties including charge, hydrophilicity and roughness. ${ }^{67}$ There were both no discernible difference in RMS surface roughness (Fig. 4g-l) and zeta potential values (Fig. $6 \mathrm{c}$ ) for the feed solution $\mathrm{pH} \approx 7$ between SG-0.3 and SG-0 surfaces. Therefore, the lower fouling propensity of the SG-0.3 membrane is ascribed to the significant enhancement in hydrophilicity, which could drastically weaken the hydrophobic interaction between hydrophobic BSA molecule and membrane surface. ${ }^{29,67}$ Specifically, the newly formed hydration boundary layer with coordinated water molecules on the SG-0.3 membrane could render surface resistance to foulant adsorption. ${ }^{62}$ In summary, the attachment of SG monomer endows the membrane with low-fouling and easy-cleaning surface that tends to resist initial macromolecular adsorption and accumulation on membrane surface such as proteins and polysaccharides providing the nutrient sources for subsequent biofilm-formation microbes.
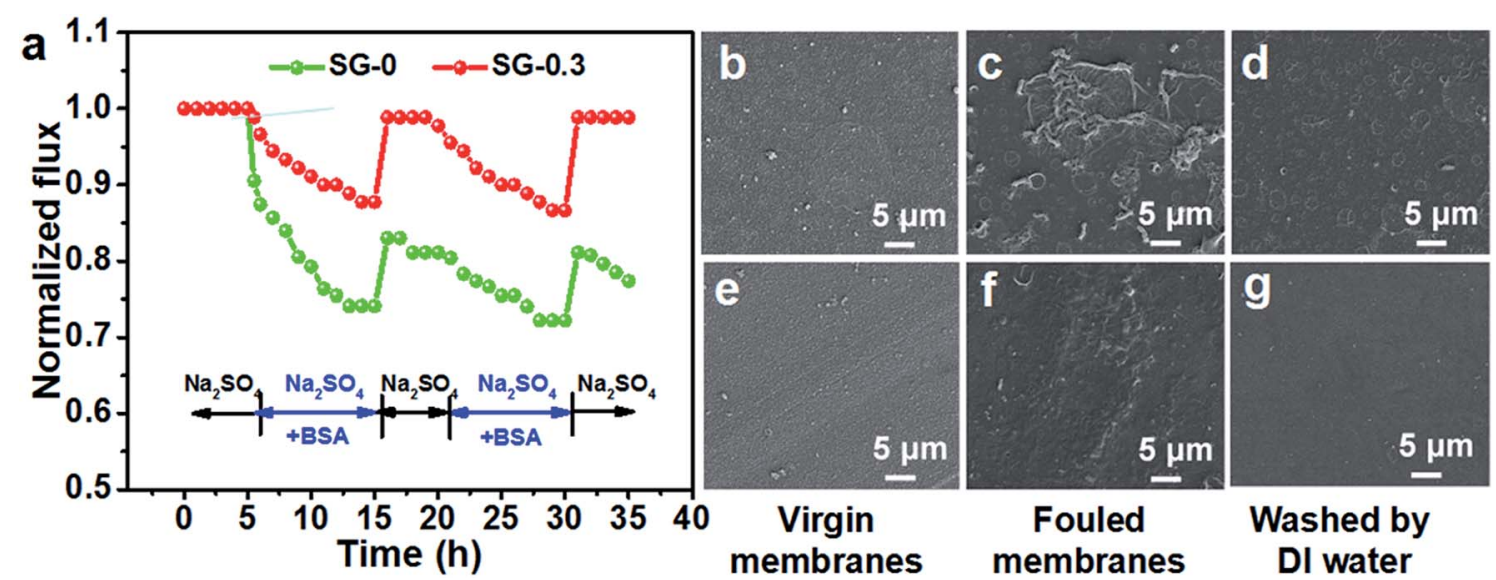

Fig. 7 (a) Time-dependent relative water flux of SG-0 and SG-0.3 membrane tested with $2 \mathrm{~g} \mathrm{~L}^{-1} \mathrm{Na}_{2} \mathrm{SO}_{4}$ and $2 \mathrm{~g} \mathrm{~L}^{-1} \mathrm{Na}_{2} \mathrm{SO}_{4}+1 \mathrm{~g} \mathrm{~L}{ }^{-1} \mathrm{BSA}$ aqueous solution at $25^{\circ} \mathrm{C}$ and 6 bar; SEM images of SG-O (b-d) and SG-0.3 (e-g) surface after antifouling experiment. 
a
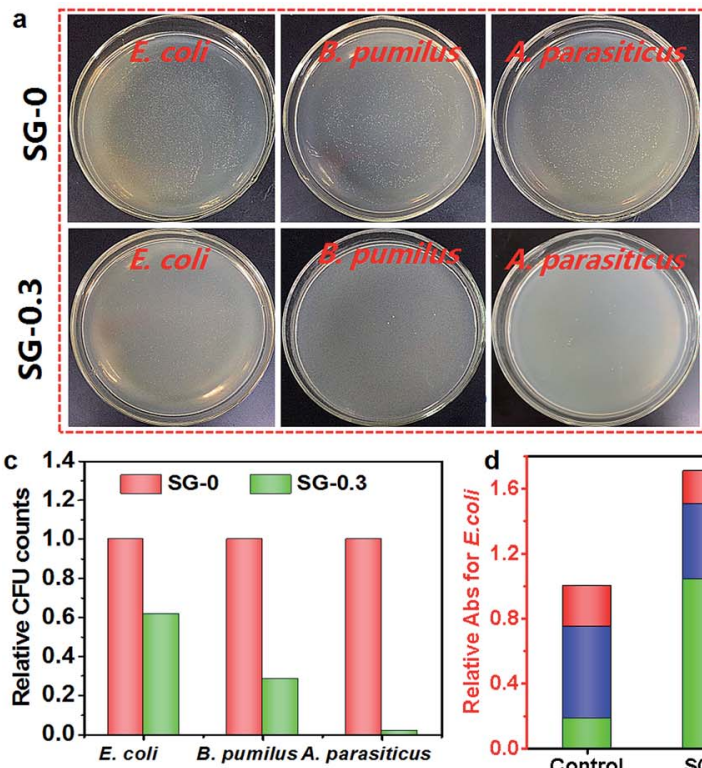
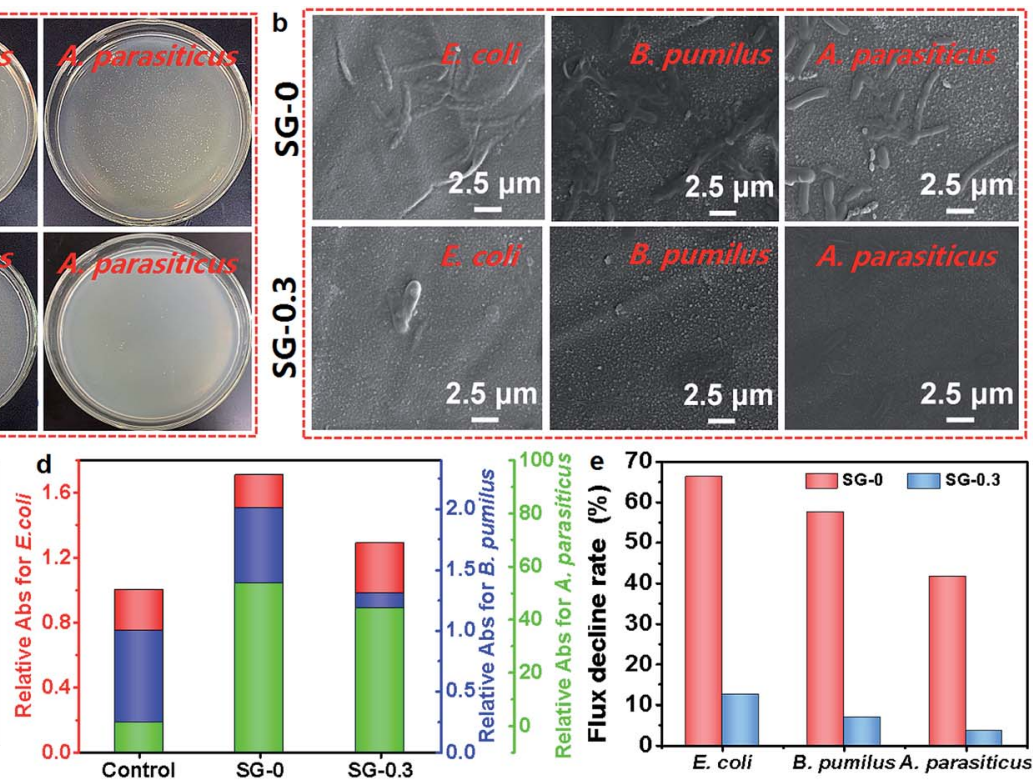

Fig. 8 (a) Photographs of three model microbes after contacting with SG-0 and SG-0.3 membranes, the E. coli and B. pumilus strains were incubated for $9 \mathrm{~h}$ at $37{ }^{\circ} \mathrm{C}$, and the A. parasiticus strain was incubated for $24 \mathrm{~h}$ at $30^{\circ} \mathrm{C}$; (b) SEM surface images of SG-0 and SG-0.3 PAs after $48 \mathrm{~h}$ exposure to $E$. coli, B. pumilus and $A$. parasiticus suspensions, respectively; (c) relative CFU counts of $E$. coli, B. pumilus and $A$. parasiticus adhering onto the SG-0 and SG-0.3 surfaces; (d) relative Abs results of $E$. coli, $B$. pumilus and $A$. parasiticus on the SG-0 and SG-0.3 surfaces; (e) water flux decline rates of SG-0 and SG-0.3 active layers after immersing into bacterial suspension.

\subsection{Membrane anti-adhesion and antimicrobial property}

For microbiological fouling studies, Gram-negative E. coli, Gram-positive B. pumilus LDS.33 and A. parasiticus JFS were chosen as the model microbes. We first evaluated the membrane anti-adhesion property of the SG-0.3 and compared that results to the SG-0. A considerable amount of accumulation of cells with full-scale adsorption were apparently observed on the SG-0 membrane, whereas the surface of SG-0.3 remained clean and only a few microbial colonies survived, as evident in photograph imaging (Fig. 8a). The result was further justified by the relative CFU value study. The lower CFU value indicates stronger microbial effects or microbial repellence (antiadhesion). The CFU of SG-0.3 membrane was dramatically reduced for all three microbes, indicating its superior resistance to microbial adhesion (Fig. 8c). Next, the antimicrobial activity of the SG-0 and SG-0.3 membranes were evaluated by growth of the microbes on it; in contrast, we took the PSf support as a control. The absorbance (Abs) of the microbial cell suspensions were detected, and the Abs value represents the number of living microbial cells after being fully contacted with membranes for a particular time. The suspension solutions without nutrients will inhibit cells multiplication and the natural death of cells is inevitable. Comparatively, the Abs value of SG-0.3 membrane was markedly lower for all three microbes, revealing the faster and more massive death of microbial cells on the SG-functionalized membrane (Fig. 8d). In addition, the NF performance of SG-0 and SG-0.3 active layers after severe biofouling was tested. The hydraulics resistance stemming from the microbes and bio-products thickly adhering onto membrane surface tend to cause flux decline, ${ }^{35}$ so the permeating fluxes of SG-0 and the SG-0.3 membrane after immersing into three microbial suspensions for $48 \mathrm{~h}$ were checked. As is shown in Fig. 8e, the flux decline rate of the SG-0 membrane for E. coli, B. pumilus, A. parasiticus dropped by approximately $66.3 \%, 57.5 \%, 41.7 \%$ after biofouling, while for the SGfunctionalized PA layer (SG-0.3), it only decreased by $12.5 \%$, $6.9 \%, 3.5 \%$, respectively, demonstrating its unprecedented antibiofouling property. The low membrane biofouling propensity of SG-0.3 was further verified by the cleaner and less damaged surface under SEM after biofouling (Fig. 8b). The results reveal that the SG-functionalized membrane holds overwhelming advantage in resisting to the microbial deposition and perturbing the growth and metabolism of microbial cells. Hence, it could be concluded that the cationic guanidinium units of the SG-functionalized membrane is responsible for the high antimicrobial activity. The positively charged SG-modified surface could strongly interact with bacterial cells containing the negatively charged phospholipid bilayers through bidentate hydrogen bonding interactions.$^{68,69}$ Besides, hydrogen bonding interactions between the keto groups of amide bonds from PA network and phospholipids might have contributed to the antimicrobial mechanism as well. ${ }^{70}$ The excellent anti-adhesion and antimicrobial properties strongly encourage us to use CASIP SG- $X$ s as a next-generation antifouling/antimicrobial membrane for practical water treatment with biofoulant species such as proteins, bacteria and cells.

\section{Conclusions}

In this work, the co-solvent assisted second interfacial polymerization (CASIP) is introduced to fabricate a highperformance SG-modified PA NF membrane with controllable thin-flim active layer and microstructures. It includes the 
addition of a synergistic acetone solvent into the water phase. As the results of FE-SEM, AFM, TEM images and gas adsorption experiments indicate, the current developed strategy could finely tune the morphology of polymeric network. The ideal size of "nanopores", and the ability to form a defect-free, thin dense selective layer led to unprecedentedly high water permeance and satisfactorily high salt rejection properties $(98.3 \%)$ for $\mathrm{Na}_{2} \mathrm{SO}_{4}$. According to the permeability tests, the flux of the CASIP SG-modified membrane enhanced remarkably (3-4 times) with no considerable salt rejection loss compared to the common PIP-TMC membrane. Besides, a series of neutral organic solution and charged dye molecule tests revealed that the membrane obtained demonstrate excellent performance in the removal of small molecules from wastewater while the longtime NF tests of CBT highlighted the superior performance stability. Finally, the resulting SG-modified PA membrane, which simultaneously possessed fouling resistance and bactericidal attributes, endowed the membrane with anti-adhesion and antimicrobial performances of high efficiency and effectiveness. Our work provides an avenue to fabricate advanced PA membranes with outstanding performance by employing the poorly water-soluble amine monomers. In consideration of the convenient and time-efficient synthetic process, it could be easily scaled-up. This attractive and promising strategy is also expected to be used in other polymeric membranes production.

\section{Conflicts of interest}

There are no conflicts to declare.

\section{Acknowledgements}

This work was supported by the National Natural Science Foundation of China (51473097), the Sichuan Science and Technology Project (2019YJ0107), and the Opening Project of State Key Laboratory of Polymer Materials Engineering (Sichuan University) (sklpme2014-3-14).

\section{References}

1 M. A. Shannon, P. W. Bohn, M. Elimelech, J. G. Georgiadis, B. J. Mariñas and A. M. Mayes, Science and Technology for Water Purification in the Coming Decades, Nature, 2008, 452(7185), 301-310.

2 M. F. Jimenez-Solomon, Q. Song, K. E. Jelfs, M. MunozIbanez and A. G. Livingston, Polymer Nanofilms with Enhanced Microporosity by Interfacial Polymerization, Nat. Mater., 2016, 15(7), 760-767.

3 M. Elimelech and W. A. Phillip, The Future of Seawater Desalination: Energy, Technology, and the Environment, Science, 2011, 333(6043), 712-717.

4 D. J. Miller, D. R. Dreyer, C. W. Bielawski, D. R. Paul and B. D. Freeman, Surface Modification of Water Purification Membranes, Angew. Chem., Int. Ed., 2017, 56(17), 4662-4711.

5 T. H. Chong, F. S. Wong and A. G. Fane, The Effect of Imposed Flux on Biofouling in Reverse Osmosis: Role of
Concentration Polarisation and Biofilm Enhanced Osmotic Pressure Phenomena, J. Membr. Sci., 2008, 325, 840-850.

6 A. Matin, Z. Khan, S. M. J. Zaidi and M. C. Boyce, Biofouling in Reverse Osmosis Membranes for Seawater Desalination: Phenomena and Prevention, Desalination, 2011, 281, 1-16.

7 Y. Han, Z. Xu and C. Gao, Ultrathin Graphene Nanofiltration Membrane for Water Purification, Adv. Funct. Mater., 2013, 23(29), 3693-3700.

8 R. Yang, J. Xu, G. Ozaydin-Ince, S. Y. Wong and K. K. Gleason, Surface-tethered Zwitterionic Ultrathin Antifouling Coatings on Reverse Osmosis Membranes by Initiated Chemical Vapor Deposition, Chem. Mater., 2011, 23, 1263-1272.

9 R. Zhang, Y. Liu, M. He, Y. Su, X. Zhao, M. Elimelech and Z. Jiang, Antifouling Membranes for Sustainable Water Purification: Strategies and Mechanisms, Chem. Soc. Rev., 2016, 45, 5888-5924.

10 J. A. Callow and M. E. Callow, Trends in the Development of Environmentally Friendly Fouling-resistant Marine Coatings, Nat. Commun., 2011, 2, 803-814.

11 A. Tiraferri, C. D. Vecitis and M. Elimelech, Covalent Binding of Single-walled Carbon Nanotubes to Polyamide Membranes for Antimicrobial Surface Properties, ACS Appl. Mater. Interfaces, 2011, 3, 2869-2877.

12 N. Misdan, A. F. Ismail and N. Hilal, Recent Advances in the Development of (Bio) fouling Resistant Thin Film Composite Membranes for Desalination, Desalination, 2016, 380, 105111.

13 D. Saeki, T. Tanimoto and H. Matsuyama, Anti-biofouling of Polyamide Reverse Osmosis Membranes Using Phosphorylcholine Polymer Grafted by Surface-initiated Atom Transfer Radical Polymerization, Desalination, 2014, 350, 21-27.

14 W. Chen, Y. Su, J. Peng, Y. Dong, X. Zhao and Z. Jiang, Engineering a Robust, Versatile Amphiphilic Membrane Surface Through Forced Surface Segregation for Ultralow Flux-decline, Adv. Funct. Mater., 2011, 21, 191-198.

15 A. M. Alswieleh, N. Cheng, I. Canton, B. Ustbas, X. Xue, V. Ladmiral, S. Xia, R. E. Ducker, O. E. Zubir, M. L. Cartron, C. N. Hunter, G. J. Leggett and S. P. Armes, Zwitterionic poly(amino acid methacrylate) brushes, J. Am. Chem. Soc., 2014, 136(26), 9404-9413.

16 J. Zhu, M. Tian, J. Hou, J. Wang, J. Lin, Y. Zhang, J. Liu and B. V. Bruggen, Surface Zwitterionic Functionalized Graphene Oxide for a Novel Loose Nanofiltration Membrane, J. Mater. Chem. A, 2016, 4(5), 1980-1990.

17 C. Dong, Z. Wang, J. Wu, Y. Wang, J. Wang and S. Wang, A Green Strategy to Immobilize Silver Nanoparticles onto Reverse Osmosis Membrane for Enhanced Antibiofouling Property, Desalination, 2017, 401, 32-41.

18 W. Ma, A. Soroush, T. V. A. Luong, G. Brennan, M. S. Rahaman, B. Asadishad and N. Tufenkji, Spray- and Spin-assisted Layer-by-layer Assembly of Copper Nanoparticles on Thin-film Composite Reverse Osmosis Membrane for Biofouling Mitigation, Water Res., 2016, 99, 188-199. 
19 J. Zhu, J. Wang, J. Hou, Y. Zhang, J. Liu and B. Van der Bruggen, Graphene-based Antimicrobial Polymeric Membranes: A Review, J. Mater. Chem. A, 2017, 5, 6776-6793.

20 G. Ye, J. Lee, F. Perreault and M. Elimelech, Controlled Architecture of Dual-functional Block Copolymer Brushes on Thin-film Composite Membranes for Integrated "Defending" and "Attacking" Strategies Against Biofouling, ACS Appl. Mater. Interfaces, 2015, 7(41), 23069-23079.

21 I. Banerjee, R. C. Pangule and R. S. Kane, Antifouling Coatings: Recent Developments in the Design of Surfaces that Prevent Fouling by Proteins, Bacteria, and Marine Organisms, Adv. Mater., 2011, 23(6), 690-718.

22 D. Rana and T. Matsuura, Surface Modifications for Antifouling Membranes, Chem. Rev., 2010, 110(4), 24482471.

23 J. Xu, Z. Wang, L. Yu, J. Wang and S. Wang, A Novel Reverse Osmosis Membrane with Regenerable Anti-biofouling and Chlorine Resistant Properties, J. Membr. Sci., 2013, 435, 80-91.

24 Y. Zhang, Z. Wang, W. Lin, H. Sun, L. Wu and S. Chen, A Facile Method for Polyamide Membrane Modification by Poly(sulfobetaine methacrylate) to Improve Fouling Resistance, J. Membr. Sci., 2013, 446, 164-170.

25 J. H. Yim, M. S. Fleischman, V. Rodriguez-Santiago, L. T. Piehler, A. A. Williams, J. L. Leadore and D. D. Pappas, Development of Antimicrobial Coatings by Atmospheric Pressure Plasma Using a Guanidine-based Precursor, ACS Appl. Mater. Interfaces, 2013, 5(22), 1183611843.

26 Y. R. Su, S. H. Yu, A. C. Chao, J. Y. Wu, Y. F. Lin, K. Y. Lu and F. L. Mi, Preparation and Properties of pH-responsive, Selfassembled Colloidal Nanoparticles from Guanidinecontaining Polypeptide and Chitosan for Antibiotic Delivery, Colloids Surf., A, 2016, 494, 9-20.

27 M. F. Ilker, K. Nüsslein, G. N. Tew and E. B. Coughlin, Tuning the Hemolytic and Antibacterial Activities of Amphiphilic Polynorbornene Derivatives, J. Am. Chem. Soc., 2004, 126, 15870-15875.

28 L. Qian, H. Xiao, G. Zhao and B. He, Synthesis of Functionalized Guanidine-based Polymers and Their Antimicrobial Activities Revealed by AFM and CLSM, ACS Appl. Mater. Interfaces, 2011, 3(6), 1895-1901.

29 T. Ishigami, K. Amano, A. Fujii, Y. Ohmukai, E. Kamio, T. Maruyama and H. Matsuyama, Fouling Reduction of Reverse Osmosis Membrane by Surface Modification via Layer-by-layer Assembly, Sep. Purif. Technol., 2012, 99, 1-7.

30 P. Gilbert and L. E. Moore, Cationic Antiseptics: Diversity of Action under a Common Epithet, J. Appl. Microbiol., 2005, 99(4), 703-715.

31 P. A. Wender, W. C. Galliher, E. A. Goun, L. R. Jones and T. H. Pillow, The Design of Guanidinium-rich Transporters and Their Internalization Mechanisms, Adv. Drug Delivery Rev., 2008, 60, 452-472.

32 S. Mitra, S. Kandambeth, B. P. Biswal, M. A. Khayum, C. K. Choudhury, M. Mehta, G. Kaur, S. Banerjee, A. Prabhune, S. Verma, S. Roy, U. K. Kharul and R. Banerjee, Self-exfoliated Guanidinium-based Ionic
Covalent Organic Nanosheets (iCONs), J. Am. Chem. Soc., 2018, 138, 2823-2828.

33 J. Nikkola, X. Liu, Y. Li, M. Raulio, H. L. Alakomi, J. Wei and C. Y. Tang, Surface Modification of Thin Film Composite RO Membrane for Enhanced Anti-biofouling Performance, $J$. Membr. Sci., 2013, 444, 192-200.

34 X. Li, Y. Cao, H. Yu, G. Kang, X. Jie, Z. Liu and Q. Yuan, A Novel Composite Nanofiltration Membrane Prepared with PHGH and TMC by Interfacial Polymerization, J. Membr. Sci., 2014, 466, 82-91.

35 Y. Gao, S. Zhao, Z. Qiao, Y. Zhou, B. Song, Z. Wang and J. Wang, Reverse Osmosis Membranes with Guanidine and Amine Enriched Surface for Biofouling and Organic Fouling Control, Desalination, 2018, 430, 74-85.

36 H. L. Zhang, Y. B. Gao and J. G. Gai, Guanidiniumfunctionalized Nanofiltration Membranes Integrating Antifouling and Antimicrobial Effect, J. Mater. Chem. A, 2018, 6(15), 6442-6454.

37 C. Kong, M. Kanezashi, T. Yamomoto, T. Shintani and T. Tsuru, Controlled Synthesis of High Performance Polyamide Membrane with Thin Dense Layer for Water Desalination, J. Membr. Sci., 2010, 362(1-2), 76-80.

38 C. Kong, T. Shintani, T. Kamada, V. Freger and T. Tsuru, Cosolvent-mediated Synthesis of Thin Polyamide Membranes, J. Membr. Sci., 2011, 384(1-2), 10-16.

39 J. Acosta-Esquijarosa, I. Rodríguez-Donis and E. PardilloFontdevila, Physical Properties and Their Corresponding Changes of Mixing for the Ternary Mixture Acetone $+n$ Hexane + Water at 298.15 K, Thermochim. Acta, 2006, 443(1), 93-97.

40 B. Khorshidi, T. Thundat, D. Pernitsky and M. Sadrzadeh, A parametric study on the synergistic impacts of chemical additives on permeation properties of thin film composite polyamide membrane, J. Membr. Sci., 2017, 535, 248-257.

41 B. Khorshidi, T. Thundat, B. A. Fleck and M. Sadrzadeh, A Novel Approach Toward Fabrication of High Performance Thin Film Composite Polyamide Membranes, Sci. Rep., 2016, 6, 22069.

42 B. Khorshidi, B. Soltannia, T. Thundat and M. Sadrzadeh, Synthesis of thin fifilm composite polyamide membranes: effect of monohydric and polyhydric alcohol additives in aqueous solution, J. Membr. Sci., 2017, 523, 336-345.

43 F. A. Pacheco, I. Pinnau, M. Reinhard and J. O. Leckie, Characterization of Isolated Polyamide Thin Films of RO and NF Membranes Using Novel TEM Techniques, $J$. Membr. Sci., 2010, 358(1-2), 51-59.

44 Y. F. Mi, Q. Zhao, Y. L. Ji, Q. F. An and C. J. Gao, A Novel Route for Surface Zwitterionic Functionalization of Polyamide Nanofiltration Membranes with Improved Performance, J. Membr. Sci., 2015, 490, 311-320.

45 C. Klaysom, S. Hermans, A. Gahlaut, S. Van Craenenbroeck and I. F. Vankelecom, Polyamide/Polyacrylonitrile (PA/PAN) Thin Film Composite Osmosis Membranes: Film Optimization, Characterization and Performance Evaluation, J. Membr. Sci., 2013, 445, 25-33.

46 X. Li, Y. Cao, H. Yu, G. Kang, X. Jie, Z. Liu and Q. Yuan, A Novel Composite Nanofiltration Membrane Prepared with 
PHGH and TMC by Interfacial Polymerization, J. Membr. Sci., 2014, 466, 82-91.

47 S. M. Xue, Z. L. Xu, Y. J. Tang and C. H. Ji, Polypiperazineamide Nanofiltration Membrane Modified by Different Functionalized Multiwalled Carbon Nanotubes (MWCNTs), ACS Appl. Mater. Interfaces, 2016, 8(29), 19135-19144.

48 M. B. M. Y. Ang, Y. L. Ji, S. H. Huang, H. A. Tsai, W. S. Hung, C. C. Hu, K. R. Lee and J. Y. Lai, Incorporation of Carboxylic Monoamines into Thin-film Composite Polyamide Membranes to Enhance Nanofiltration Performance, $J$. Membr. Sci., 2017, 539, 52-64.

49 D. Nikolaeva, C. Langner, A. Ghanem, M. A. Rehim, B. Voit and J. Meier-Haack, Hydrogel Surface Modification of Reverse Osmosis Membranes, J. Membr. Sci., 2015, 476, 264-276.

50 S. H. Huang, C. J. Hsu, D. J. Liaw, C. C. Hu, K. R. Lee and J. Y. Lai, Effect of Chemical Structures of Amines on Physicochemical Properties of Active Layers and Dehydration of Isopropanol through Interfacially Polymerized Thin-film Composite Membranes, J. Membr. Sci., 2008, 307(1), 73-81.

51 L. Ruangchuay, J. Schwank and A. Sirivat, Surface Degradation of $\alpha$-Naphthalene Sulfonate-doped Polypyrrole during XPS Characterization, Appl. Surf. Sci., 2002, 199(14), 128-137.

52 Y. Gao, S. Zhao, Z. Qiao, Y. Zhou, B. Song, Z. Wang and J. Wang, Reverse Osmosis Membranes with Guanidine and Amine Enriched Surface for Biofouling and Organic Fouling Control, Desalination, 2018, 430, 74-85.

53 E. M. Hoek, S. Bhattacharjee and M. Elimelech, Effect of Membrane Surface Roughness on Colloid-Membrane DLVO Interactions, Langmuir, 2003, 19(11), 4836-4847.

54 P. Veerababu, B. B. Vyas, P. S. Singh and P. Ray, Limiting Thickness of Polyamide Polysulfone Thin-film-composite Nanofiltration Membranes, Desalination, 2014, 346, 19-29.

55 R. Dawson, A. I. Cooper and D. J. Adams, Nanoporous Organic Polymer Network, Prog. Polym. Sci., 2012, 37(4), 530-563.

56 N. B. McKeown and P. M. Budd, Exploitation of Intrinsic Microporosity in Polymer Based Materials, Macromolecules, 2010, 43(12), 5163-5176.

57 T. Wang, Y. Yang, J. Zheng, Q. Zhang and S. Zhang, A Novel Highly Permeable Positively Charged Nanofiltration Membrane Based on a Nanoporous Hyper-crosslinked Polyamide Barrier Layer, J. Membr. Sci., 2013, 448, 180-189.

58 B. Liang, P. Zhang, J. Wang, J. Qu, L. Wang, X. Wang, C. Guan and K. Pan, Membranes with Selective Laminar
Nanochannels of Modified Reduced Graphene Oxide for Water Purification, Carbon, 2016, 103, 94-100.

59 J. Sekulic, J. E. ten Elshof and D. H. A Blank, A Microporous Titania Membrane for Nanofiltration and Pervaporation, Adv. Mater., 2004, 16(17), 1546-1550.

60 K. Y. Wang and T. S. Chung, The Characterization of Flat Composite Nanofiltration Membranes and Their Applications in the Separation of Cephalexin, J. Membr. Sci., 2005, 247(1-2), 37-50.

61 M. Vazda, P. Jungwirth and P. E. Mason, Aqueous Guanidinium-Carbonate Interactions by Molecular Dynamics and Neutron Scattering: Relevance to Ionprotein Interactions, J. Phys. Chem. B, 2013, 117(6), 18441848.

62 L. Shen, X. Zhang, J. Zuo and Y. Wang, Performance Enhancement of TFC FO Membranes with Polyethyleneimine Modification and Post-treatment, $J$. Membr. Sci., 2017, 534, 46-58.

63 C. Gao, Hydrophilic Modification of Pervaporation Membrane and Intensification of Water Transport Process, Doctoral dissertation, Tianjin University, 2015.

64 J. Wang, Z. Wang, J. Wang and S. Wang, Improving the Water Flux and Bio-fouling Resistance of Reverse Osmosis (RO) Membrane through Surface Modification by Zwitterionic Polymer, J. Membr. Sci., 2015, 493, 188-199.

65 J. Schaep, B. Van der Bruggen, C. Vandecasteele and D. Wilms, Influence of Ion Size and Charge in Nanofiltration, Sep. Purif. Technol., 1998, 14, 155-162.

66 H. Fan, J. Gu, H. Meng, A. Knebel and J. Caro, High-Flux Membranes Based on the Covalent Organic Framework COF-LZU1 for Selective Dye Separation by Nanofiltration, Angew. Chem., Int. Ed., 2018, 57(15), 4083-4087.

67 E. M. Vrijenhoek, S. K. Hong and M. Elimelech, Influence of Membrane Surface Properties on Initial Rate of Colloidal Fouling of Reverse Osmosis and Nanofiltration Membranes, J. Membr. Sci., 2001, 188(1), 115-128.

68 D. Y. Sasaki and T. M. Alam, Solid-state 31P NMR Study of Phosphonate Binding Sites in Guanidine-functionalized, Molecular Imprinted Silica Xerogels, Chem. Mater., 2000, 12(5), 1400-1407.

69 J. Yatvin, J. Gao and J. Locklin, Durable Defense: Robust and Varied Attachment of Non-leaching Poly“-onium" Bactericidal Coatings to Reactive and Inert Surfaces, Chem. Commun., 2014, 50(67), 9433-9442.

70 A. Kozubek and J. H. Tyman, Resorcinolic Lipids, the Natural Non-isoprenoid Phenolic Amphiphiles and Their Biological Activity, Chem. Rev., 1999, 99(1), 1-25. 